\title{
L'apport de la science-fiction aux discours technoscientifiques sur la conquête de Mars
}

\section{The contribution of science fiction to technoscientific discourses on the} conquest of Mars

\author{
Thomas Michaud ${ }^{1}$ \\ ${ }^{1}$ Docteur en sciences de gestion, Chercheur indépendant, France, michaud.thomas@yahoo.fr
}

RÉSUMÉ. La conquête de Mars est un enjeu qui dépasse le cadre de la curiosité scientifique. Si l'exploration est essentiellement produite et financée par le secteur public, l'envoi de missions habitées dans le but de créer une civilisation martienne pourrait être assuré par des acteurs privés. La science-fiction s'est inspirée de ces discours pour proposer un imaginaire dont la fonction est étudiée dans cet article. Après avoir examiné l'apport de ce genre à l'élaboration des discours de la Mars Society et de l'entrepreneur Elon Musk, cette étude s'interroge sur les modalités de construction des micro-discours idéologiques, comme le marsisme. Ce néologisme désigne le passage des imaginaires science-fictionnels aux idéologies technologiques, particulièrement influentes dans la dynamique du capitalisme. Interroger l'imaginaire est important pour stimuler la R\&D, et pour assurer la fusion des intérêts et attentes des ingénieurs, des innovateurs, et du public.

ABSTRACT. The conquest of Mars is an issue that goes beyond the scope of scientific curiosity. Although exploration is mainly produced and financed by the public sector, the deployment of manned missions with the aim of creating a Martian civilization could be provided by private actors. Science fiction was inspired by these discourses to propose an imaginary whose function is studied in this article. After examining the contribution of this genre to the elaboration of the speeches of the Mars Society and of the entrepreneur Elon Musk, this study questions the construction of ideological micro-discourses such as Marsism. This neologism refers to the passage from science fiction to technological ideologies, particularly influential in the dynamics of capitalism. Exploring the imaginary is important for stimulating R\&D and for merging the interests and expectations of engineers, innovators and the public.

MOTS-CLÉS. Science-fiction, marsisme, idéologies, technotypes, innovation, ingénieurs, planète Mars.

KEYWORDS. Science fiction, marsism, ideologies, technotypes, innovation, engineers, planet Mars.

\section{Introduction}

Parmi les multiples utopies technologiques qui alimentent le capitalisme global, la conquête de Mars et la création d'une civilisation sur cette planète apparait comme une des plus ambitieuses [CHI 92]. Cette planète est présentée tantôt comme un enfer hostile à toute forme de vie, tantôt comme un lieu d'implantation rêvé pour quelques scientifiques, entrepreneurs et ingénieurs à la recherche de nouvelles frontières à explorer et à domestiquer. Le secteur spatial est un des plus innovants, générant des retours sur investissements importants et utiles au progrès de la civilisation humaine. Si les grandes agences spatiales ont longtemps eu le monopole de l'accès à l'espace, de nouveaux modèles économiques émergent depuis les années 2010. Nommé New Space, ce courant est principalement défini par l'intérêt de milliardaires et d'entrepreneurs pour exploiter l'espace, par le tourisme, l'exploitation d'astéroïdes ou la création de bases extraterrestres conçues comme les prémisses d'une expansion de l'humanité dans le système solaire. Il ne fait presque aucun doute que la conquête de l'espace sera au centre d'une prochaine révolution industrielle. Etudier l'imaginaire qui anticipe ce processus depuis plus d'un siècle est important. En effet, quel est l'impact de la science-fiction sur la construction des discours stratégiques des innovateurs, et sur l'imaginaire des ingénieurs et scientifiques dont la fonction implicite est de réaliser les rêves les plus fous de l'humanité ? [FLI 01] [FLI 95] Comment fonctionne cet imaginaire technique? [HOT 13] Quel est son rôle dans l'élaboration des idéologies technologiques, et est-il raisonnable de penser que les technologies utopiques de la science-fiction ont pour vocation à être réalisées par les acteurs de la Recherche et Développement (R\&D)? 
Cet article s'intéresse à l'apport de la science-fiction dans la construction des discours sur la conquête et la colonisation de la planète Mars [BOI 87]. Les récits sur une éventuelle civilisation martienne ont suscité au dix-neuvième siècle de nombreuses spéculations et récits à la frontière de l'astronomie et d'une science-fiction émergente. Il fallut attendre les années 1960 et la sonde Mariner 4 pour que les premières photographies de cette planète dévoilent un paysage aride, probablement sans vie et hostile à l'humain, ce qui étonna la communauté scientifique. Si de nombreuses dystopies ont vu le jour depuis quelques années, nous retiendrons l'influence de certaines fictions sur des scientifiques, utopistes et entrepreneurs motivés par la conquête de cette planète. Nous présenterons quelques éléments du discours de Robert Zubrin, scientifique qui a créé la Mars Society et publié plusieurs ouvrages qui ont contribué à populariser l'idée d'envoyer des missions habitées vers Mars dans les prochaines décennies. Son livre le plus connu, Cap sur Mars, ainsi que son roman On a marché sur Mars ont contribué à populariser l'idée d'envoyer une mission habitée vers Mars depuis les années 1990. Très influencé par la science-fiction, et notamment par l'idée de terraformation, sujet d'une trilogie de Kim Stanley Robinson [ROB 12], il envisage les modalités pratiques d'une implantation durable de l'humanité sur Mars, et les différentes étapes permettant de créer les conditions de l'habitabilité de cette planète pour l'homme. Son utopisme a largement contribué à alimenter les discours les plus positifs à une époque lors de laquelle l'envoi d'humains sur Mars n'était pas raisonnable budgétairement, et surtout technologiquement, la mise au point de vaisseaux nécessitant encore des innovations majeures pour assurer le bon déroulement de ce type de missions. Les arguments de Zubrin étaient-il pris au sérieux par la communauté scientifique dans les années 19902000, ou faisait-il figure d'illuminé, fasciné par des visions du futurs science-fictionnelles très éloignées des considérations pragmatiques des investisseurs? [DIS 00].

Dans un deuxième temps, l'article s'intéressera au discours futuriste du milliardaire Elon Musk. Bien connu au départ de sa carrière pour avoir fait fortune dans le secteur des Technologies de l'Information et de la Communication (TIC), il développe de nombreux projets technologiques qui pourraient changer les conditions de vie de l'humanité à moyen terme. Son projet principal est l'envoi d'hommes sur Mars dans le but d'y développer une ville d'un million d'habitants. Musk propose un discours très influencé par la science-fiction et n'hésite pas à injecter des thèmes imaginaires dans ses discours. Cela a pour effet d'intéresser de nombreux acteurs avides d'aventures et de challenges technoscientifiques. Musk intervient par exemple sur les dangers de l'intelligence artificielle, sur la création de l'hyperloop, un train supersonique, et sur la neuroconnexion, qui appartenait jusqu'alors à certains films et romans cyberpunks. Il s'intéresse aussi aux voitures autonomes, et à bien d'autres thèmes qui tendent à sortir de la pure science-fiction à mesure que des découvertes et des innovations apparaissent, promettant de réaliser les rêves les plus fous [EAS 08].

\section{L’imaginaire martien de la Mars Society}

L’Association Planète Mars (Mars Society) a été créée en 1998 par Robert Zubrin. Son but est de promouvoir les missions martiennes auprès des grandes agences comme la National Aeronautics and Space Administration (NASA). Zubrin est un ingénieur connu pour avoir rédigé des articles influents, présentant un plan pour envoyer des hommes sur Mars. Le plan Mars Direct a notamment été développé dans le livre Cap sur Mars. Depuis, la Mars Society a ouvert des antennes dans de nombreux pays, notamment en Europe, entretenant un lobbying international pour la concrétisation du rêve martien. Si de nombreux scientifiques ont soutenu cette initiative, des auteurs de science-fiction comme Kim Stanley Robinson ou James Cameron ont aussi manifesté leur intérêt. Conquérir Mars est présenté comme un enjeu stratégique. De nombreux membres de l'association s'intéressent à la science-fiction, conçue comme un moyen d'expression de représentations du futur, mais aussi comme un mode de communication publicitaire de la cause martienne [GEO 00] [DUN 13]. 
Sur son blog ${ }^{1}$, Pierre Brisson, président de la section suisse de la Mars Society présente quelques œuvres de science-fiction, rédigées par des membres de l'association. Un de ses articles est intitulé «Utopie, science-fiction, fantasy ; l'imagination est le moteur de notre action ». Il évoque le roman de Richard Heidmann [HEI 17], polytechnicien, diplômé de l'Ecole Nationale Supérieure de l'Aéronautique et ancien directeur à la Société nationale d'étude et de construction de moteurs d'aviation (SNECMA), un des concepteurs des moteurs d'Ariane. L'action d'Alerte à Mars City se déroule en 2143. Un journaliste se rend sur Mars, où une colonie compte 50000 membres. Pierre Brisson évoque aussi un roman de Jean Marc Salotti, professeur des universités en informatique à l'Ecole Nationale de Cognitique de l'Institut Polytechnique de Bordeaux, secrétaire du groupe de travail de l'Académie Internationale d'Astronautique chargé de faire le point sur les missions martiennes habitées et d'établir une liste de recommandations pour les agences spatiales internationales, et également membre du Conseil de direction de l'Association Planète Mars. Cet expert présente dans Genèse martienne [SAL 16] les difficultés que rencontreront probablement les premiers explorateurs de cette planète. Deux types d'œuvres de science-fiction martienne se distinguent. Certaines traitent des difficultés de l'exploration, et d'autres du fonctionnement de sociétés, voire d'une civilisation humaine sur Mars [DEW 03].

La hard science fiction développe des récits le plus réaliste possible. Robert Zubrin a ainsi proposé dans On a marché sur Mars [ZUB 06] un récit visant à éclairer par la fiction son plan d'exploration martienne. La Mars Society est devenue en quelques années un lobby puissant, particulièrement influent, qui cherche à convaincre les grandes agences ou d'éventuels investisseurs à la recherche de l'esprit pionnier qui anime l'humanité et plus particulièrement le peuple américain. La rédaction de romans de science-fiction par certains de ses membres montre l'influence de cet imaginaire dans les communautés scientifiques ou d'ingénieurs, particulièrement dans le secteur spatial [CH0 15].

Lors de la vingtième convention annuelle internationale de la Mars Society, en septembre 2017, un débat donnait la parole à des auteurs de science-fiction prestigieux dont le but était de définir le rôle de cette discipline dans l'élaboration des visions et des stratégies des pionniers de l'exploration et de la colonisation de Mars. Gregory Benford faisait partie des auteurs invités. Il avait notamment préfacé le roman de Robert Zubrin [ZUB 06] et a rédigé des fictions à succès comme Les enfants de Mars [BEN 01]. David Brin est aussi une référence, auteur de plusieurs romans à succès et membre du NASA's advisory board on Innovative and Advanced Concepts Group. Jerry Pournelle et Larry Niven participaient aussi au débat.

La science-fiction est une matrice imaginaire très présente dans les débats scientifiques et chez les décideurs dans le secteur spatial [JOH 11]. Associer les citoyens aux missions exploratoires est une nécessité pour les entrepreneurs à la recherche de budgets permettant de financer leurs projets. Utiliser la science-fiction pour communiquer avec le public est une méthode bien connue, au moins aux EtatsUnis, depuis que la série Star Trek a accompagné et stimulé les ingénieurs et scientifiques des missions d'exploration lunaire [WES 98] [WES 00]. La science-fiction est un stimulant puissant de l'imaginaire, un élément discursif tellement mobilisateur que certains lobbies comme la Mars Society y ont recours pour participer à la planification de projets prometteurs pour la conquête et la colonisation de Mars. Au niveau des grandes agences spatiales, l'intérêt pour la science-fiction est une évidence. L'Agence Spatiale Européenne (ESA) a produit le rapport Innovative Technologies From Science Fiction (ITSF), une étude répertoriant les apports majeurs de la science-fiction aux innovations techniques révolutionnaires ou à venir dans les technosciences utiles à la conquête spatiale. La NASA organise aussi régulièrement des concours de science-fiction, notamment autour du projet martien.

https://blogs.letemps.ch/pierre-brisson/2017/08/15/utopie-dystopie-science-fiction-fantasy-limagination-est-le-moteur-de-notreaction/ 
L'imaginaire martien est-il nécessaire ou un frein à l'innovation, la profusion des nouvelles références n'ayant toujours pas été accompagnée par une mission habitée réussie sur Mars ? Les films et romans ne risquent-ils pas d'effrayer le public, potentiellement choqué par certaines histoires ? Dans le film Seul sur Mars (2015) par exemple, le héros est abandonné sur une planète hostile suite à une gigantesque tempête, ses coéquipiers ayant quitté la base pour revenir sur Terre avoir l'avoir considéré comme mort. La critique a unanimement salué le réalisme du film, qui a bénéficié de l'aide de plusieurs scientifiques avisés sur les sujets comme la survie sur Mars, l'agronomie ou la climatologie. David Mimoun, spécialiste de Mars et professeur à Supaero, affirmait que «Seul sur Mars est une excellente pub pour le métier d'ingénieur » en 2016. La science-fiction peut aussi avoir un effet négatif face à certaines innovations en diffusant un imaginaire négatif, peu mobilisateur, voire même effrayant. D'autres films mettent en scène des martiens hostiles, comme dans Ghosts of Mars, ou des conditions de survie très difficiles. Le spationaute martien est bien souvent une tête brûlée, conscient de participer à une tâche historique et prêt à risquer sa vie pour être parmi les premiers humains à poser le pied sur Mars.

Robert Zubrin est convaincu de la nécessité de diffuser un imaginaire martien positif dans la société. Le coût financier et humain d'une mission habitée exige la popularité et l'adhésion d'un grand nombre de citoyens. Une agence qui engagerait inconsciemment des sommes considérables dans une mission impopulaire risquerait sa réputation et un scandale majeur en cas d'échec de la mission et de mort d'un ou de plusieurs membres du vaisseau.

Nous pouvons constater des mutations de l'imaginaire technique à mesure que les connaissances scientifiques évoluent. Dans le secteur martien, un grand nombre de fictions ont évoqué les martiens alors qu'aucune connaissance rationnelle sur cette planète n'était vraiment disponible. La fin des illusions mena l'imaginaire vers une réflexion à la fois imaginaire et pragmatique. Il fallait évaluer les conditions de réalisation d'une première mission de pionniers, comme pour contribuer à une réflexion technocientifique nécessitant des avancées importantes au niveau de la R\&D. D'autres œuvres, plus rares, décrivent un futur plus lointain, dans lequel une base martienne a fini par générer une colonie, voire une civilisation. Ce grand projet excite l'imaginaire, et est au fondement de pratiques politiques futures conditionnées par des découvertes majeures de la part des scientifiques et des ingénieurs dans les prochaines décennies. Notons cependant que la science-fiction est rarement produite par des décideurs et politiciens. Les scientifiques sont cependant de très grands lecteurs et producteurs de science-fiction, qui est devenue un élément de dialogue et de communication entre les inventeurs et les décideurs [MCC 97].

Penser le futur des missions martiennes par le lobbying n'est pas une chose aisée. La Mars Society de Robert Zubrin a souvent rencontré des difficultés pour convaincre de la nécessité de financer rapidement une mission de pionniers. Pour l'instant, les décideurs n'ont pas décidé de réaliser le rêve martien. Zubrin a pourtant présenté dans Cap sur Mars un projet détaillé de mission habitée, chiffré et argumenté en fonction de la connaissance dans les années 1990-2000. Son roman illustre ce plan, et les dangers et intérêts pour l'humanité de se rendre sur Mars. Depuis les années 2010, la conquête de Mars a pris une autre voie. La mission habitée de la NASA n'étant pas d'actualité, des ambitions privées émergèrent, et notamment par l'intermédiaire d'un savant entrepreneur, Elon Musk, milliardaire féru de science-fiction et pour lequel rien ne semble impossible. Il s'inspira d'ailleurs des idées de la Mars Society au début des années 2000 , avant de profiter de l'élan collectif lancé par certains milliardaires ayant fait fortune dans la nouvelle économie. 


\section{Elon Musk, un esprit d'entrepreneur utopiste}

Elon Musk est un entrepreneur très médiatique qui a fait fortune en créant Pay $\mathrm{Pal}^{2}$, et qui a depuis investi dans plusieurs secteurs technologiques comme l'automobile et les lanceurs spatiaux. Sa formation en physique et en commerce est accompagnée de nombreuses lectures, notamment de science-fiction. Lors de différents entretiens, il a indiqué avoir particulièrement apprécié le cycle de Fondation, d'Isaac Asimov, en raison de la théorie des cycles historiques qui explique la croissance et le déclin des civilisations. Cette idée l'a incité à envisager une colonisation du système solaire afin d'éviter la fin de l'humanité. Il plaça dans sa voiture Tesla envoyée dans l'espace en 2018 les romans d'Asimov, conférant une dimension symbolique à cette initiative. Elon Musk rapporte aussi avoir été influencé par le roman de Robert Heinlein Révolte sur la Lune, publié en 1966, pendant la préparation du programme Apollo. Cette fiction imagine la colonisation de la Lune. Musk y a puisé l'idée de créer une base extraterrestre, particulièrement utile pour définir son projet de civilisation martienne. Le livre Le guide du voyageur galactique, de Douglas Adams, contribua selon lui à gérer une crise existentielle traversée lors de l'adolescence. Il serait exagéré de considérer que la science-fiction a intégralement guidé la vision stratégique de ce milliardaire américain. Il est en effet doté d'une grande culture et se nourrit de multiples lectures, dans différents secteurs, comme les biographies, ou les ouvrages scientifiques.

Elon Musk est un entrepreneur visionnaire. Il maîtrise une communication visant à faire partager ses rêves les plus délirants au plus grand nombre. Annoncer la création prochaine d'une civilisation multiplanétaire appartenait avant Musk au monde de l'imaginaire, de la science-fiction, et de la prospective technoscientifique [PRA 98]. Peu d'acteurs du secteur spatial évoquaient l'arrivée prochaine de technologies suffisamment viables et de capitaux importants pour envoyer une mission habitée vers Mars. Musk annonce qu'il réalisera cet exploit, et qu'il créera une base, voire une colonie sur la planète rouge en un temps record. Cet article s'intéresse essentiellement à l'impact de la science-fiction sur les ingénieurs et entrepreneurs mobilisés dans l'exploration et la conquête de Mars. Il faut aussi consulter ses multiples déclarations pour constater qu'il envisage des scénarios de science-fiction pour projeter dans un avenir hypothétique les conséquences civilisationnelles, organisationnelles et sociétales d'innovations technologiques qu'il met au point avec des équipes fascinées par un discours managériale enjôleur. La science-fiction est érigée au rang de discours fédérateur, autant pour les ingénieurs travaillant dans les différentes entreprises de Musk, que pour des clients qui, en achetant des produits d'Elon Musk, ont l'impression de participer à un rêve collectif dont les racines culturelles et imaginaires se trouvent chez des auteurs comme Asimov ou Heinlein [WEI 95]. Musk joue avec son image de technophile génial, imprégné par la culture geek, pour orienter les représentations collectives de l'avenir dans les communautés de chercheurs, notamment aux Etats-Unis. Il cultive cette image, au point que de nombreux fans et médias le considèrent comme le réel Iron Man. Ce superhéros est un milliardaire qui a mis au point de nombreuses innovations comme une armure surpuissante lui permettant de lutter contre les forces du mal.

Elon Musk a diffusé un discours science-fictionnel qui participe à la création d'une bulle spéculative autour de ses activités. S'il n'est pas un superhéros Marvel, il est devenu un superentrepreneur, héros d'une génération d'ingénieurs et d'innovateurs qui rêvent de disposer de son aura et de sa puissance financière. Chaque génération cultive des représentations idéales-typiques des entrepreneurs. Pensons par exemple à Henry Ford ou Bill Gates, qui sont devenus de véritables mythes grâce à leur approche révolutionnaire du business et du management.

\footnotetext{
${ }^{2}$ Pay Pal est une entreprise créée en 1998 par plusieurs fondateurs dont Elon Musk. Il s'agit d'un service de paiement en ligne qui a rencontré un grand succès au point d'être rachetée par eBay en 2002, faisant la fortune de ses inventeurs. En 2017, Paypal détenait $80 \%$ des parts de marché des paiements électroniques.
} 
Il n'en reste pas moins que le rêve martien a reçu de nombreuses critiques, notamment de la part de Kim Stanley Robinson, qui estime que le plan de Musk « ressemble à un gros cliché de science-fiction des années 1920, imaginé par un garçon qui tente de construire une fusée pour atteindre la Lune depuis son jardin à l'arrière cours ». Musk n'est pas le seul milliardaire à investir dans la conquête spatiale. Le mouvement du New Space voit depuis la décennie 2010 des centaines d'acteurs privés s'intéresser à l'exploitation $\mathrm{du}$ cosmos, conçu comme une nouvelle frontière potentiellement source de profits considérables pour les entrepreneurs les plus audacieux. Jeff Bezos, fondateur d'Amazon, s'inscrit dans la ligne du discours spatial de Musk. Plutôt que Mars, il s'intéresse à la colonisation de la Lune. La Terre ne peut pas continuer à abriter l'industrie lourde, selon lui, car elle est trop polluante et dangereuse. Dans les prochaines décennies, il souhaite transférer ces activités dans l'espace, et plus précisément sur la Lune. En mars 2017, il a même présenté à Donald Trump (Président des Etats-Unis depuis 2017) un projet de vaisseau spatial permettant d'effectuer des livraisons sur la Lune. Quelques mois plus tard, Trump annonça sa volonté d'envoyer des hommes sur la Lune pour préparer une mission martienne, assurant la synthèse des rêves de Bezos et Musk, dans une perspective étatique, la NASA étant, rappelons le, à l'origine de la plupart des rêves spatiaux d'une nation américaine fascinée par la science-fiction.

Le président de l'agence spatiale française, le Centre National d'Etudes Spatiales (CNES), JeanYves Le Gall, a été interrogé par plusieurs revues à la fin 2017 pour faire partager sa vision des prochaines missions vers Mars. Il a soutenu dans Challenges une conception "réaliste" de la conquête spatiale, opposée aux "projets de science-fiction", défendus notamment par Elon Musk :

«Aujourd'hui, personne ne croit au show d'Elon Musk. La présentation qu'il a faite l'année dernière au Mexique, à Guadalajara, était un show hollywoodien. Il y avait de superbes images de synthèse, mais personne ne croit qu'un vaisseau va partir sur Mars en embarquant une centaine de personnes, qui plus est pour un aller simple. Je réfute totalement la comparaison qui est faite avec le peuplement de l'Amérique. Le peuplement de l'Amérique, vous aviez trois semaines de bateau, et si vous en réchappiez, il y avait des prairies, des dindons, des cours d'eau douce, on pouvait construire une maison et s'installer. Vous allez sur Mars, il n'y a pas d'atmosphère, il n'y a rien. Vous dépendez uniquement du vaisseau qui vous a amené. Et l'histoire d'aller extraire des minerais, de l'eau, ce n'est même pas futuriste, c'est au delà de la science-fiction. Il faut savoir raison garder $»^{3}$.

Pour Jean-Yves Le Gall, il faudra entre 100 et 200 ans pour réaliser la vision d'Elon Musk, qu'il assimile à un délire de science-fiction. Le réalisme français contraste avec une conception sciencefictionnelle de l'exploration et de la conquête des acteurs américains les plus influents. Cette dichotomie est révélatrice des différences d'approches entre ces 2 cultures. Le Gall est même tenté d'assimiler le discours de Musk à une forme d'irrationalité, alors que ce dernier envisage d'envoyer une mission habitée vers Mars dès 2024, soit seulement 7 ans après la critique de Le Gall. L'avenir dira si l'approche science-fictionnelle, américaine, de Musk, aura triomphé, ou si le réalisme français a eu raison de promouvoir une approche plus prudente d'une ambition probablement moins bien diffusée dans l'imaginaire collectif. La mentalité de pionnier du peuple américain est aussi probablement plus réceptive des concepts science-fictionnels, que la société française, voire européenne.

\section{Le technotype de la base extraterrestre}

L'exploration martienne est passée depuis quelques années dans une nouvelle ère, avec l'émergence de nouveaux acteurs, pour certains privés, envisageant l'implantation de colonies humaines sur cette planète dans le but de créer une nouvelle civilisation, voire une nouvelle forme de commerce

${ }^{3}$ https://www.challenges.fr/entreprise/aeronautique/conquete-de-mars-personne-ne-croit-au-show-hollywoodien-d-elon- 
interplanétaire. La science-fiction fascine depuis longtemps les scientifiques et ingénieurs des grandes agences spatiales, comme la NASA et l'ESA. Des concours de science-fiction ayant pour thème la présence de l'humanité dans l'espace sont régulièrement organisés pour associer les citoyens, notamment les plus jeunes, à la R\&D spatiale. Cette dernière est une recherche de pointe, souvent à l'origine d'innovations majeures ayant des retombées importantes sur la vie quotidienne de milliards d'humains. La science-fiction est un élément récurrent dans la recherche appliquée. Si la recherche fondamentale, plus théorique, est difficilement liable à cet imaginaire, le travail d'investigation sur les applications du futur des découvertes scientifiques est en revanche proche de la science-fiction, constituant même une forme de science-fictionnalisation institutionnelle. Cette formule désigne la pratique d'imagination de futures applications ou procédés dans le cadre d'agences ou d'institutions, comme des entreprises ou des centres de R\&D étatiques. La science-fiction constitue bien souvent une sous-culture inhérente à certaines organisations, dans les secteurs les plus innovants [MIN 16]. Les découvertes dans le secteur spatial cherchent bien souvent à réaliser la science-fiction. Voyager vers la Lune revient à concrétiser les rêves d'illustres romanciers comme Savinien de Cyrano de Bergerac, auteur d'une Histoire comique des Etats et empires de la lune et du soleil (1657 et 1662) ou Jules Verne, qui a publié le prophétique De la Terre à la Lune en 1865.

La révolution industrielle a besoin d'un imaginaire technique pour alimenter la dynamique du capitalisme qui en est à la fois la cause et la conséquence [MAR 95]. La science-fiction est particulièrement active dans les périodes de forte innovation, faisant craindre à certains qu'elle génère des catastrophes, s'il était avéré que la $R \& D$ réalise la science-fiction. De nombreux récits mettent en effet en scène des mondes dystopiques, ou des évènements dans lesquels la civilisation humaine est soumise à rude épreuve. L'imaginaire apocalyptique de la science-fiction est une réactualisation de schèmes imaginaires ancestraux, qui dirigent de nombreuses sociétés depuis longtemps. La technoscience est conçue à la fois comme la cause de catastrophes menant à l'anéantissement de la civilisation, et comme la possibilité pour l'humanité de survivre et de contrôler la nature.

De plus, la science-fiction se situe bien souvent dans le futur. Cette spécificité, qui appartenait jusqu'alors aux oracles, n'est que rarement assumée par des auteurs qui ne souhaitent pas être présentés comme une nouvelle forme de prophètes. Là encore, il faut remonter loin dans l'histoire pour avoir l'explication d'une telle posture intellectuelle [MIN 96]. Longtemps, les prédictions des oracles furent dénoncées car elles ne provenaient pas de Dieu mais des demons [CAZ 86]. Il fallait donc s'en méfier et préférer les discours de l'Eglise à des prédictions parfois assimilées à une forme de sorcellerie, dangereuses pour l'ordre établi. La science-fiction est apparue après que ces considérations religieuses ont subi les foudres révolutionnaires, notamment en Europe. Penser le futur devint une nouvelle liberté, souvent appréciée par les citoyens, surtout lorsqu'elle décrivait des sociétés dans lesquelles le progrès permettait de révolutionner les modes de vies, grâce notamment au positivisme [CHE 12]. La sciencefiction devint progressivement un genre très populaire, faisant rêver les masses, en s'inspirant des découvertes scientifiques pour proposer un nouvel imaginaire, dans une certaine mesure programmatique pour une communauté scientifique de plus en plus érigée en classe dominante, surtout après la Seconde Guerre mondiale. Réaliser l'imaginaire devint un leitmotiv permettant l'avènement d'une ère reposant sur l'innovation et la R\&D [SFE 02] [DRE06].

Dans ces conditions, la conquête de Mars faisait partie de ce programme imaginaire, au même titre que la télévision, l'informatique, la quête de l'immortalité, etc. La base extraterrestre, qu'elle soit lunaire ou martienne, émergea progressivement comme un nouvel imaginaire, comme un technotype, c'est à dire un archétype technologique dévoilé par l'innovation et le progrès technoscientifique. La science-fiction généra des représentations futuristes ${ }^{4}$ de ce technotype, alimentant la communauté

\footnotetext{
${ }^{4}$ Le site technovelgy, qui recense plus de 2500 inventions techniques issues d'œuvres de science-fiction, indique que la première évocation de base lunaire date de 1930, dans le roman Brigands of the Moon, de Ray Cummings. La formule Moon Dome était alors 
scientifique en nouveaux projets et challenges. Dès lors, l'imaginaire s'est emparé de ce technotype pour proposer de multiples applications de ce système technique récemment conscientisé par l'humanité [DUR 93] [GOL 95]. Les premiers auteurs à aborder l'exploration et la conquête de Mars ne disposaient que de données scientifiques partielles de cette planète. Les spéculations sur une éventuelle civilisation martienne étaient fréquentes et admises dans de nombreuses sociétés savantes. Les premières missions américaines envoyées vers Mars montrèrent qu'il n'existait pas de civilisation. Mars est un désert, probablement hostile à l'humanité, et il faudrait des efforts considérables pour rendre cette planète accueillante. L'imaginaire martien connut alors un temps d'arrêt. Les nouvelles œuvres firent preuve d'une nouvelle forme d'utopisme avec, dans les années 1990, la parution de la trilogie de Kim Stanley Robinson (Mars la rouge, Mars la bleue, Mars la verte) présentant la terraformation de Mars comme un nouveau défi pour les scientifiques. Il envisageait l'implantation de colonies humaines sur Mars, dans le but d'en modifier le climat pour rendre la planète vivable pour l'humanité. La terraformation était un concept déjà présent dans la communauté scientifique dans les années 1960. Mais Robinson parvint à faire de son œuvre un nouveau mythe sectoriel, permettant de fédérer la communauté scientifique autour de nouvelles représentations futuristes [MOL 90]. Le but n'est plus seulement de contacter des martiens, souvent craints, d'ailleurs, dans les œuvres de sciencefiction du dix-neuvième et du début du vingtième siècle. Il faut désormais orienter les recherches vers de nouvelles trajectoires. Il ne s'agit pas de préparer une éventuelle guerre contre les martiens, mais d'apprendre à manipuler le climat d'une planète pour la rendre vivable pour l'humanité.

\section{Marsisme, nasaisme, communicisme, des micro-idéologies technoscientifiques}

L'imaginaire science-fictionnel s'inspire des découvertes scientifiques qu'il confronte à des problématiques sociétales souvent teintées d'idéologie [MAN 06] [RIC 05]. La fusion des sciences dures et des sciences de la société génère cet imaginaire, influent dans les communautés scientifiques autant que dans une population profane, contribuant pourtant indirectement à l'élaboration des politiques de R\&D et au financement de la science. La rencontre de la science et de la démocratie est facilitée par la science-fiction, véritable interface entre la fiction et la réalité. Les systèmes imaginaires issus de ce processus d'extrapolation et d'exploration des possibles futurs technoscientifiques génèrent des micro-idéologies alimentant par la suite les grandes idéologies technologiques, émergeant d'une forme d'underground. Avant de décrire le passage de l'imaginaire science-fictionnel à la microidéologie technoscientifique, présentons quelques exemples de cette dernière.

Le Marsisme est une micro-idéologie technoscientifique dans la mesure où l'imaginaire martien suscite un intérêt dans les communautés de chercheurs au point de générer des financements, voire même d'orienter les processus de prise de décision au niveau managérial et politique [SFE 95]. Initialement, l'imaginaire martien est partiellement inspiré par les découvertes scientifiques. Il permet aussi leur dépassement, une appropriation collective, populaire, voire démocratique de ses thématiques. La micro-idéologie marsiste désigne le passage de l'imaginaire dans une nouvelle étape de la réflexion collective. Les principales œuvres, dont certaines comme la trilogie martienne de Robinson constituent des mythes sectoriels, sont un point d'horizon vers lequel tend une grande partie des chercheurs et innovateurs. Terraformer Mars n'est pas une possibilité à court terme. Il n'en reste pas moins que l'élaboration des plans des missions à long terme est influencée par cette science-fiction. Dans un premier temps, un film comme Seul sur Mars, plébiscité par la critique pour son réalisme, est un modèle pour les futurs astronautes qui seront envoyés en mission vers cette planète. La science-fiction génère des récits qui contribuent à la formation des ingénieurs. Il serait erroné de considérer cette contribution exagérément, au point de sous-estimer l'influence majeure des disciplines scientifiques dans leurs formations. La micro-idéologie joue cependant un rôle d'interface entre trois acteurs

utilisée. Le terme Moon Base fut utilisé pour la première fois en 1948, à la fois par L. Ron Hubbard dans 240,000 Miles Straight Up et par Robert Heinlein dans Space Cadet. 
majeurs du processus de prise de décision et d'innovation. Le scientifique, le décideur-entrepreneur, et le public assimilent à des moments divers les discours science-fictionnels, ce qui mène parfois à la constitution d'idéologies technologiques influentes à un niveau global.

Le Communicisme est un autre exemple de micro-idéologie technoscientifique particulièrement active depuis l'avènement d'Internet. Dans ce cas, nous disposons de plus de recul qu'avec le marsisme dans la mesure où Internet est devenu une réalité technologique, ce qui n'est pas le cas de la base martienne. Si les auteurs de science-fiction ont depuis le milieu du dix-neuvième siècle anticipé les principales innovations dans le secteur de la télévision et de l'informatique en réseau, il faut considérer que le courant cyberpunk, à partir des années 1980, a accompagné la mise en place d'Internet. Il serait cependant dommage de considérer que la science-fiction n'a pas prédit Internet, comme l'affirment la plupart des commentateurs. Les cyberpunks et les écrivains des années 19802020 n'ont eu de cesse d'imaginer le devenir d'Internet sous sa forme future, c'est-à-dire une simulation globale immersive, considérée elle aussi comme un technotype émergent. Si l'idéologie de la communication décrite par Lucien Sfez [SFE 88] [SFE 02] ou l'utopie de la communication étudiée par Philippe Breton [BRE 00] permettent de comprendre les phénomènes idéologiques et utopiques sous-jacents à l'émergence de cette technologie, nous estimons que la science-fiction a joué un rôle particulier dans la construction de cette idéologie-utopie de la communication. Breton a d'ailleurs consacré un passage entier de son livre Le culte d'Internet à l'étude du roman d'Asimov Face aux feux $d u$ soleil [BRE 00] pour illustrer le fonctionnement de l'utopie de la communication. Dans le cas d'Internet et du virtuel, la micro-idéologie nommée communicisme désigne la contribution de la science-fiction à la création d'un climat collectif facilitant, parfois exagérément, voire d'une manière délirante, le financement de politiques publiques ou privées de la R\&D de ce secteur. La sciencefiction permet dans ce cas l'adhésion d'une grande quantité d'acteurs qui travaillent collectivement à l'avènement et à l'interprétation du technotype de la simulation globale immersive.

Le Nasaisme est un troisième néologisme qui désigne une micro-idéologie technoscientifique, cette fois-ci institutionnelle [PAR 92]. La NASA produit en effet un grand nombre de discours scientifiques et évite de créer une confusion dans le public, rétablissant parfois la réalité lorsque des discours peu rationnels tentent de tromper les citoyens avec de fausses informations. La NASA joue le rôle de régulateur de l'imaginaire technoscientifique et organise parfois des concours de science-fiction pour stimuler l'imaginaire de millions d'adeptes de conquête spatiale dans le monde. Ce type de structure, de même que l'ESA (European Space Agency) avec l'Esaisme, proposent ce que Lucien Sfez nomme dans La Santé Parfaite des «fictions sciences » [SFE 95] dont la fonction est d'assurer une forme de sens commun vis-à-vis de la connaissance scientifique et de proposer des explications sur l'origine et l'avenir de l'espèce humaine dans une très longue histoire, cosmique. Les découvertes de la NASA alimentent de nombreux fantasmes, ainsi que des récits de science-fiction depuis sa création en 1958. Elle peut aussi être conçue comme la conséquence d'une ébullition science-fictionnelle aux Etats-Unis depuis plusieurs décennies [LUO 13].

Selon quelles modalités l'imaginaire science-fictionnel devient-il une micro-idéologie technoscientifique [MAL 00] ? Dans un premier temps, l'imaginaire apparait chez des auteurs aux références variables et protéiformes. Plutôt que de parler d'un inconscient collectif commun à tous les auteurs et à tous les humains, à ce niveau initial, il semble que les schèmes imaginaires apparaissent chez des individus isolés, bien que vivant dans une culture à la fois spécifique et globale. Dans un second temps, certains de ces récits tombent dans l'oubli, d'autres touchent un public plus large alors que les plus aboutis sont élevés au rang de mythe sectoriel. On pense au cyberespace de William Gibson, et à la terraformation de Kim Stanley Robinson. A ce niveau, une sélection s'effectue dans la somme des récits, même si tous contribuent à proposer des technologies utopiques, manifestations imaginaires de technotypes que les innovateurs sont chargés de conscientiser et de matérialiser sous la forme de nouvelles technologies commercialisables. Nous avons pu analyser dans des publications précédentes comment l'imaginaire du virtuel avait donné naissance au communicisme, une micro- 
idéologie qui a finalement pu muter sous la forme d'une idéologie technologique particulièrement influente. Dans le cas de l'imaginaire science-fictionnel martien, nous avons constaté sa mutation sous la forme d'une micro-idéologie, le marsisme, en raison de l'appropriation par des acteurs scientifiques et économiques de ses thématiques. Ce phénomène explique en partie la croyance selon laquelle la science-fiction serait dotée d'un pouvoir de précognition. En fait, ces fictions contribuent à l'innovation à mesure qu'elles sont insérées dans des discours idéologiques provenant d'acteurs de plus en plus proches des processus de décision politique et économique. Un cercle vertueux est constaté en ce qui concerne l'influence de la science-fiction chez les décideurs. Dans un premier temps, une grande partie d'entre eux a baigné dans une culture très imprégnée par cet imaginaire depuis plusieurs décennies. La science-fiction télévisuelle a notamment contribué à propager sur toute la planète une forme d'utopisme technologique provenant principalement des Etats-Unis. Dans un second temps, ces derniers ont continué à créer une science-fiction qu'ils estimaient importante pour orienter la Recherche et Développement à un niveau global. Progressivement, manager et innover devint un synonyme de réaliser un imaginaire technique de plus en plus formaté et idéologique. De nombreuses fictions institutionnelles émanant d'entreprises, ou d'agences spatiales comme la NASA ou l'ESA ont expliqué au grand public que l'imaginaire était important pour innover et que la science-fiction pouvait être une source d'inspiration, mais aussi un mode de communication, de storytelling, particulièrement mobilisateur. Lorsque la NASA et l'ESA présentent leurs programmes, leurs vidéos sont à la frontière de la science-fiction et du documentaire. De nombreux films de prospective d'entreprise suivent d'ailleurs la même démarche. L'esthétique science-fictionnelle est devenue une référence pour les acteurs chargés de planifier le futur, de convaincre les citoyens consommateurs de la qualité de la société utopique qu'ils souhaitent réaliser.

Dans le secteur spatial, de nouveaux acteurs émergent chaque jour. Adeptes du New Space, ils souhaitent libéraliser l'accès à l'espace, concurrencer les organisations technocratiques telles que la NASA. Ils adhéraient souvent à un imaginaire science-fictionnel datant pour certains de plusieurs décennies. Coloniser est un instinct primordial de l'espèce humaine. Il est donc évident que certains acteurs, parfois nommés « astropreneurs », consacrent une partie de leur fortune pour être des pionniers de l'ultime frontière et passer à la postérité. Les bases sur des territoires extraterrestres sont des technotypes que la science-fiction a contribué à cartographier et à représenter. La phase du New Space promet le passage du marsisme, jusqu'alors utilisé et véhiculé dans des lobbies comme la Mars Society ou les grandes agencies, comme par exemple la NASA ou l'ESA, à une autre dimension. Les prospectivistes du New Space attendent une révolution technologique, notamment dans le secteur de la propulsion et des moteurs, pour que le marsisme devienne une idéologie technologique majeure. L'exploitation de certains astéroïdes ou d'éventuels métaux sur Mars, pourrait générer une économie d'un nouveau type et un capitalisme interplanétaire.

\section{Conclusion}

L'histoire de l'exploration de la planète Mars a montré l'influence des découvertes scientifiques dans l'imaginaire technopolitique. Au dix-neuvième siècle, on pensait qu'une civilisation martienne avait construit des réseaux de canaux, laissant même certains observateurs inquiets d'une éventuelle hostilité de ces voisins méconnus. La science-fiction contribua à diffuser cette idée [ALK 10]. Elle devint en effet un imaginaire populaire très apprécié aux Etats-Unis pendant l'entre deux guerres. Une erreur scientifique de l'astronome Schiaparelli engendra un imaginaire collectif foisonnant, suscitant le désir de mieux connaitre ce peuple extraterrestre. La science fut par la suite à l'origine de la découverte de l'inexistence des martiens. Pendant plusieurs décennies, la science fut la source d'une irrationalité collective. La science-fiction fut particulièrement riche à cette époque, témoignant d'un imaginaire généré par une théorie erronée. Les énormes progrès réalisés dans la connaissance de cette planète ont provoqué une mutation de l'imaginaire, qui oriente désormais l'humanité vers son expansion interplanétaire. Dans le même temps, l'imaginaire devint un élément important de l'élaboration des 
politiques de R\&D et des stratégies. Le capitalisme ne craint plus une éventuelle guerre contre des martiens supérieurs technologiquement. Désormais, il s'inspire de la science-fiction, présentée comme une source d'utopies technologiques utile au management des organisations et de la société [SEG 05].

\section{Bibliographie}

[ADM 10] ADAMS D., H2G2: L'intégrale de la trilogie en cinq volumes, Denoël, Paris, 2010.

[ALK 10] ALKON P.K., Origins of Futuristic Fiction, University of Georgia Press, 2010.

[BEN 01] BENFORD G., Les enfants de Mars, Presses de la Cité, Paris, 2001.

[BOI 87] BOIA L., L’Exploration imaginaire de l'espace, La Découverte, Paris, 1987.

[BRE 00] BRETON P., Le culte de l'Internet, une menace pour le lien social?, La Découverte, Paris, 2000.

[CAZ 86] CAZES B., Histoire des futurs, les figures de l'avenir de Saint-Augustin au XXIème siècle, Seghers, Paris, 1986.

[CHE 12] CHENG J., Astounding Wonder: Imagining Science and Science Fiction in Interwar America, University of Pennsylvania Press, 2012.

[CHI 92] CHIRPAZ F., Raison et déraison dans l'utopie, L'Harmattan, Paris, 1992.

[CHO 15] CHOUTEAU M., FAUCHEUX M., NGUYEN C., "Les élèves ingénieurs à l'épreuve de la fiction. Quelles relations entretiennent-ils avec les mondes scientifiques et techniques fictionnels ?", Les Enjeux de l'information et de la communication, disponible à: http://lesenjeux.u-grenoble3.fr/, no. 16/3A, 2015.

[CYR 12] CYRANO DE BERGERAC S., Histoire comique des états et empires de la Lune et du Soleil, Hachette Livre BNF, Paris, 2012.

[DEW 03] DE WITT D.K., Astrofuturism: Science, Race and Visions of Utopia in Space, University of Pennsylvania Press, 2003.

[DIS 00] DISCH T., The Dreams Our Stuff Is Made Of: How Science Fiction Conquered the World, Free Press, New York, 2000.

[DRE 06] DREGNI E., DREGNI J., Follies of Science - 20th Century Visions of Our Fantastic Future, Speck Press, Denver, CO, 2006.

[DUN 13] DUNNE A., RABY F., Speculative Everything: Design, Fiction, and Social Dreaming, MIT Press, London, 2013.

[DUR 93] DURAND G., Les structures anthropologiques de l'imaginaire, Dunod, Paris, 1993.

[EAS 08] EASTON L., SCHROEDER R., The Influence of Imagination: Essays on Science Fiction and Fantasy as Agents of Social Change, McFarland \& Company, 2008.

[FLI 95] FLICHY P., L'innovation technique: Récents développements en sciences sociales, vers une nouvelle théorie de l’innovation, La Découverte, Paris, 1995.

[FLI 01] FLICHY P., L’imaginaire d'Internet, La Découverte, Paris, 2001.

[GEO 00] GEOFF K., Science Fiction Cinema: From Outerspace to Cyberspace, Wallflower, London, 2000.

[GOL 95] GOLDEN K.L., Science Fiction, Myth, and Jungian Psychology, The E. Mellen Press, Lewiston, 1995.

[HEI 17] HEIDMANN R., Alerte à Mars City - La colonie Martienne comme si vous y étiez, Editions Auteurs d'Aujourd'hui, 2017.

[HOT 13] HOTTOIS G., Généalogies philosophique, politique et imaginaire de la technoscience, Vrin, Paris, 2013.

[JAM 07] JAMESON F., Archéologie du futur, un désir nommé utopie, Max Milo, Paris, 2007.

[JAM 08] JAMESON F., Archéologie du futur, penser la science-fiction, Tome 2, Max Milo, Paris, 2008.

[JOH 11] JOHNSON B.D., Science Fiction for Prototyping: Designing the Future with Science Fiction, Morgan \& Claypool Publishers, 2011.

[LUO 13] LUOKKALA B.B., Exploring Science through Science Fiction, Springer Science \& Business Media, 2013.

[MAL 00] MALRIEU P., La construction des imaginaires, L'Harmattan, Paris, 2000. 
[MAN 06] MANNHEIM K., Idéologie et utopie, Ed. de la Maison des sciences de l'homme, DL, Paris, 2006.

[MAR 95] MARCUS G.E., Technoscientific Imaginaries, University of Chicago Press, Chicago, IL, 1995.

[MCC 97] MCCURDY H., Space and the American Imagination, Smithsonian Institution Press, Washington, DC, 1997.

[MIN 96] MINOIS G., Histoire de l'avenir, des prophètes à la prospective, Fayard, Paris, 1996.

[MIN 16] MINVIELLE N., WATHELET O., MASSON A., Jouer avec les futurs: utilisez le design fiction pour faire pivoter votre entreprise, Pearson Education, Paris, 2016.

[MOL 90] MOLES A., «La fonction des mythes dynamiques dans la construction de l'imaginaire social », Cahiers de l'imaginaire, no. 5/6, pp. 9-33, 1990.

[PAR 92] PARKER M. (ed.), Utopia and Organization, Blackwell, Oxford, 1992.

[PRA 98] PRANTZOS N., Voyages dans le futur, l'aventure cosmique de l'humanité, Le Pommier, Paris, 1998.

[RIC 05] RICOEUR P., L'idéologie et l'utopie, Seuil, Paris, 2005.

[ROB 12] ROBINSON K.S., La Trilogie martienne, Omnibus, Paris, 2012.

[SAL 16] SALOTTI JM., Genèse martienne Tome 1 Objectif Mars, Amalthée, 2016.

[SEG 05] SEGAL H.P., Technological Utopianism in American Culture, Syracuse University Press, New York, 2005.

[SFE 88] SFEZ L., Critique de la Communication, Le Seuil, Paris, 1988.

[SFE 95] SFEZ L., La santé parfaite, critique d'une nouvelle utopie, Seuil, Paris, 1995.

[SFE 02] SFEZ L., Technique et idéologie, un enjeu de pouvoir, Seuil, Paris, 2002.

[VER 01] VERNE J., De la Terre à la Lune, Le livre de Poche, Paris, 2001

[WEI 95] WEICK K.E., Sensemaking in Organizations, Sage Publications, Thousand Oaks, CA, 1995.

[WEI 15] WEIR A., Seul sur Mars, Bragelonne, Paris, 2015

[WES 98] WESTFAHL G., The Mechanics of Wonder: The Creation of the Idea of Science Fiction, Liverpool University Press, 1998.

[WES 00] WESTFAHL G., Space and Beyond: The Frontier Theme in Science Fiction, Greenwood Publishing Group, 2000.

[ZUB 04] ZUBRIN R., Cap sur Mars: un plan pour l'exploration et la colonisation de Mars par l'homme, Editions Goursau, Paris, 2004.

[ZUB 06] ZUBRIN R., On a marché sur Mars, Presses de la cite, Paris, 2006. 